\title{
Zu Beginn einer CPAP-Therapie steigt das Gewicht
}

Bourey RE et al. Early gain in body mass with continuous positive airway pressure

therapy for obstructive sleep apnea. Somnologie 2010;14:207-212

\section{Hintergrund und Fragestellung}

Bei Patienten mit einem obstruktiven Schlafapnoesyndrom (OSAS) wurden wenige Monate nach einer CPAP-Therapie sowohl eine Gewichtszunahme als auch eine Gewichtsabnahme beschrieben. Neue Studien weisen darauf hin, dass die durch die CPAP-Therapie ausgelösten Veränderungen eine Umverteilung des Körperfetts bewirken. Bei konstantem Gewicht wird anscheinend vor allem das viszerale Fett reduziert.

In einer aktuellen retrospektiven Studie wurde das Gewicht von OSAS-Patienten vor und vier Wochen nach einer CPAPTherapie untersucht und interessante pathophysiologische Erklärungen für die initiale Gewichtszunahme gefunden.

\section{Methodik}

Der Gewichtsverlauf von 152 konsekutiven Patienten wurde analysiert. Vor und vier Wochen nach einer CPAP-Therapie (29 Tage) wurden die Polysomnografiedaten, der Apnoe-Hypopnoe-Index (AHI), der Body-Mass-Index (BMI), der Blutdruck, die Herzfrequenz und der Schläfrigkeitsscore (Epworth Sleepiness Scale, ESS) ermittelt.

\section{Ergebnisse}

Die tägliche Nutzungszeit der CPAPTherapie betrug 5,9 Stunden. Nach der einmonatigen Behandlung wurde bei $78 \%$ der Patienten (81\% der Männer und $73 \%$ der Frauen) eine Gewichtszunahme beobachtet (1,4 kg; Abb. 1).

Eine Korrelation zwischen der Gewichtszunahme und den gemessenen Parametern wie der CPAP-Nutzungsdauer fand sich nicht.

In einer Untergruppe von 71 Patienten, von denen die Daten auch nach sechs Monaten (193 Tage) zur Verfügung standen, fand keine weitere Gewichtszunahme statt.

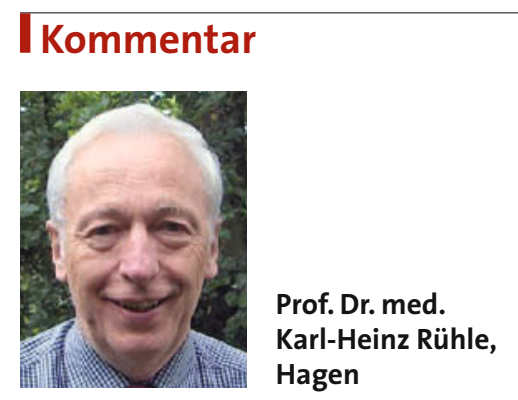

Nach der genannten und weiteren Studien scheint es durch die CPAP-Therapie schon innerhalb weniger Tage zu einer Gewichtszunahme zu kommen. Diese schnelle Gewichtszunahme wurde bei einigen Patienten schon nach vier Tagen beobachtet.

Würde die Gewichtszunahme allein auf eine vermehrte Kalorienzufuhr zurückzuführen sein, würde dies eine zusätzliche Aufnahme von 500 Kalorien/Tag bedeuten, die sehr unwahrscheinlich ist. Vielmehr kommt eine andere Erklärung infrage: Durch die CPAP-Therapie steigt das vaskuläre Blutvolumen infolge einer verbesserten endothelialen Funktion, einer verminderten sympathischen Aktivität und einer verminderten Urinausscheidung an.
Es ist ein bekanntes Phänomen, dass unter der CPAP-Therapie die nächtliche Urinausscheidung zurückgeht und das Plasmavolumen erhöht wird. Durch die verbesserte Schlafarchitektur wird die Plasma-Renin-Aktivität verdoppelt und der Aldosteronspiegel um 50\% erhöht. Außerdem wird das atriale natriuretische Peptid (ANP) unter der CPAP-Therapie reduziert. Alle genannten Mechanismen erhöhen das vaskuläre Blutvolumen.

Es konnte gezeigt werden, dass bereits nach einer Nacht die Urinproduktion um die Hälfte von 504 auf $269 \mathrm{ml}$ reduziert und das Plasmavolumen um 12,7\% erhöht wurde. Schätzt man das Plasmavolumen auf drei Liter, dann würde die Veränderung der Urinausscheidung schon nach einer Nacht zu einer Zunahme des Gewichts um o,4 kg führen.

\section{Fazit}

Die initial beobachtete Gewichtszunahme von etwa 1,4 $\mathrm{kg}$ bei OSAS-Patienten nach dem Beginn einer CPAP-Therapie dürfte nicht auf eine gesteigerte Kalorienzufuhr, sondern auf die positiven neurohumoralen Effekte der Überdruckbeatmung zurückzuführen sein.

Man würde den OSAS-Patienten, die eine diätetische Gewichtsreduktion durchführen wollen, Unrecht tun, wenn man ihnen wegen der Gewichtszunahme Diätfehler unterstellen würde. Bei einer unveränderten Kalorienzufuhr könnte die kurzfristige Zunahme des BMI sogar als integrales Maß für den günstigen Effekt der CPAPTherapie hinsichtlich der kardiovaskulären Funktionen gewertet werden.

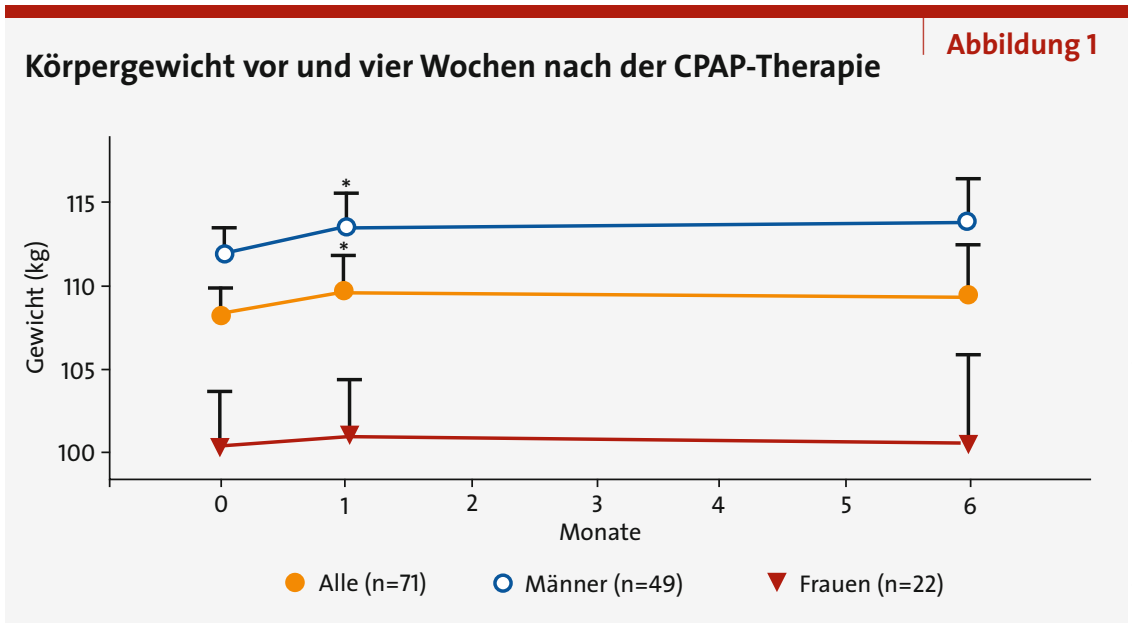

